\title{
The inhibitory effect against collagen-induced arthritis by Schistosoma japonicum infection is infection stage-dependent
}

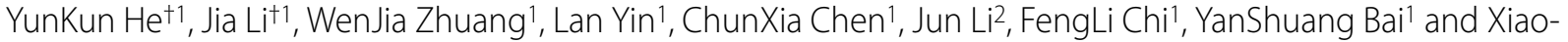 \\ Ping Chen*1
}

\begin{abstract}
Background: A long-term existing schistosome infection can aid in maintaining immuno-homeostasis, thus providing protection against various types of autoimmune diseases to the infected host. Such benefits have often been associated with acute or egg stage infection and with the egg-induced Th2 response. However, since schistosome infection undergoes different stages, each associated with a specific induction of Th responses, the requirements for the ability of the different stages of schistosome infection to protect against autoimmune disease has not been elucidated. The present study was designed to study whether different stages of schistosome infection offer unique protection in collagen-induced arthritis and its mechanisms.

Results: Arthritis susceptible strain DBA/1 male mice were infected with Schistosoma japonicum for either 2 weeks resulting in early stage infection or for 7 weeks resulting in acute or egg stage infection. Following Schistosoma japonicum infection, collagen II was administered to induce collagen-induced arthritis, an animal model for human rheumatoid arthritis. Infection by Schistosoma japonicum significantly reduced the severity and the incidence of experimental autoimmune collagen-induced arthritis. However, this beneficial effect can only be provided by a preestablished acute stage of infection but not by a pre-established early stage of the infection. The protection against collagen-induced arthritis correlated with reduced levels of anti-collagen II IgG, especially the subclass of IgG2a. Moreover, in protected mice increased levels of IL-4 were present at the time of collagen II injection together with sustained higher IL-4 levels during the course of arthritis development. In contrast, in unprotected mice minimal levels of IL-4 were present at the initial stage of collagen II challenge together with lack of IL-4 induction following Schistosoma japonicum infection.

Conclusion: The protective effect against collagen-induced arthritis provided by Schistosoma japonicum infection is infection stage-dependent. Furthermore, the ability of schistosomiasis to negatively regulate the onset of collageninduced arthritis is associated with a dominant as well as long-lasting Th2 response at the initiation and development of autoimmune joint and systemic inflammation.
\end{abstract}

\section{Background}

The increased incidences of autoimmune diseases and atopic diseases found in developed countries [1,2] have brought the 'hygiene hypothesis' into a hot area of study and debate. The 'hygiene hypothesis' was first proposed by the British scientist Dr. Strachan in 1989 after having

\footnotetext{
*Correspondence: xc9718@hotmail.com

1 Department of Immunology, Tongji University School of Medicine, 1239

Siping Road, Shanghai 200092, China

+ Contributed equally

Full list of author information is available at the end of the article
}

observed that having many siblings, especially older ones, correlated with a decreased risk of hay fever [3]. This finding has since been extended to a theory that the changed pattern in or the reduced exposure to microorganisms has led to a dysregulated immune system and hence led to increases in certain disorders like atopy and autoimmune diseases. Indeed, the mutual exclusion relationship between the incidence of immune-mediated disorders with some kinds of microbes infections, especially parasite infections, has repeatedly been reported in epi- 
demiological studies and in animal models[4,5]. However, the requirement of the nature of parasite infection has not been fully elucidated.

Worm-like metazoan organisms so called 'helminth', including both nematoda (round worms) and platyhelminthes (flatworms), have been recognized as important infectious agents that can elicit beneficial effects to the infected host in terms of conferring resistance to atopy or autoimmune diseases. As a representative genus in parasitic platyhelminthes, schistosome or exposure to schistosome derived antigens have been found to offer protection to a range of autoimmune disorders in experimental animal models including type 1 diabetes in nonobese diabetic (NOD) mice [6,7], experimental allergic encephalomyelitis (EAE) (an animal model of multiple sclerosis) [8,9], Graves' disease [10], inflammatory bowel disease [11] and asthma [12]. However, the effect of helminth infection on collagen-induced arthritis, an animal model for human rheumatoid arthritis (RA), is less-well studied[13,14].

The immune response elicited by Schistosoma mansoni $(\mathrm{Sm})$, the species that is mostly seen in Africa and South America, progresses through two phases. During the first 2-5 weeks, called early stage infection, in which the host is exposed to migrating immature parasites, the dominant response is Th1. As the parasites mature, mate and begin to produce eggs, the infection enters the acute stage during which the Th1 response decreases and the Th2 response emerges and increases. The Th2 response decreases after 12 weeks of chronic stage of the infection $[15,16]$. Similar immune response profiles are also found in Schistosoma Japonicum (Sj), the species mostly present in Asia [17,18].

Majority of animal studies have found that the protective effects against immune-mediated disorders provided by schistosome infection appeared to be associated with Th2 immune response induced at egg-stage or acute stage of infection. Only one study done by Osade et al on collagen-induced arthritis (CIA) model has demonstrated that the early stage of schistosome infection might exert any beneficial effects [14]. They found that protective effects against CIA in mice can be provided by 2 weeks $S m$ infection [14], an early stage of $S m$ infection in which eggs have not been produced in large quantities and a Th2-dominant response is usually not seen [19]. This observed protection against CIA offered at early-stage infection lacking association with a Th2 response prompted us to question whether different stages of schistosome infection would offer unique protection and whether a Th2-dominanted cytokine milieu provided by egg-stage of schistosome infection was required to achieve protective effects in CIA model. Answers to these questions will help us to better understand the mecha- nisms involved in parasite immune defense and use them to prevent and treat autoimmune arthritis.

In this study, we selected either a 2 or 7 weeks $S j$ infection as early or acute stages of infection to study whether different stages of $S j$ infection would affect the development of CIA differently. We found that only the 7 weeks infection regimen can offer protective or prophylactic effects against CIA, whereas the 2 weeks infection failed to provide any beneficial effect and even exacerbated the disease. Further studies indicated that the protective effects were correlated with decreased levels of anti-collagen II IgG especially the IgG2a subclass. Cytokine pattern analysis indicated that the presence of the Th2 cytokine milieu at the initiation period of CIA together with its sustained high levels was critical for the acquirement of protective effects.

\section{Results}

The protective effects against arthritis were only observed when a pre-established $\mathrm{Sj}$ infection entered acute stage; whereas an early stage of $\mathrm{Sj}$ infection exacerbated the arthritis

$S m$ infection can alleviate the development of several autoimmune diseases like type I diabetes [6,7] and experimental autoimmune encephalomyelitis $[8,9]$ when the infection enters the acute stage or egg-stage of infection and when a Th2 response is dominant. To determine whether different stages of schistosomiasis would affect uniquely autoimmune arthritis in CIA model, the most widely accepted animal model for human rheumatoid arthritis (RA), and the role played by a pre-established dominant Th2 environment, DBA/1 mice were infected with the parasitic worm $S j$ either for 2 weeks for preestablished early stage of $S j$ infection (ESCIA, low Th2 response) or for 7 weeks for a pre-established acute stage of $S j$ infection (ASCIA, Th2 dominant) before the induction of CIA by collagen II (CII) immunization.

As shown in Fig. 1, a significantly induced Th2 response as evaluated by a significant increase in IL-4 production by polyclonally activated spleen $\mathrm{T}$ cells was found in the 7 weeks infection state as compared to the 2 weeks infection state and to mice not infected. However, a slightly reduced Th1 cytokine IFN- $\gamma$ production was observed in mice infected with 7 weeks compared to those infected with 2 weeks and those with no infection but without any statistical significance. Thus, consistent with the profiles found in other strains of mice infected by $S m$ or by $S j$ [16-18], DBA/1 mice also displayed Th2 dominant response when the infection enters 7 weeks but not the 2 weeks.

Arthritis was induced by application of collagen in complete Freund's adjuvant (CFA) followed by incomplete Freund's adjuvant (IFA) 21 days after first CII injection. Similar as reported [20], DBA/1 mice developed 
A

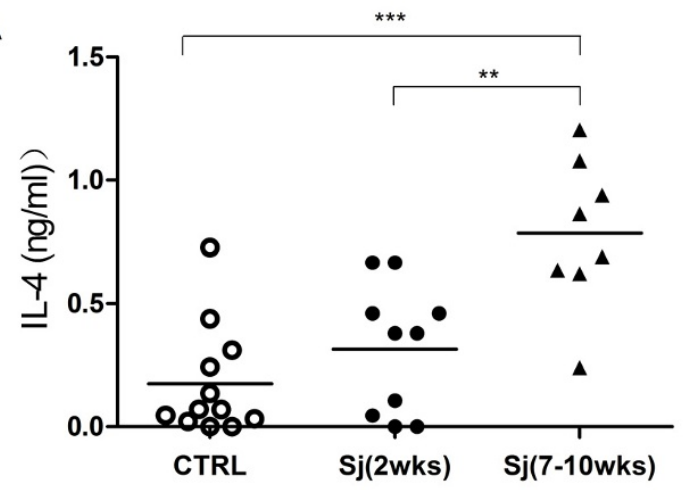

B

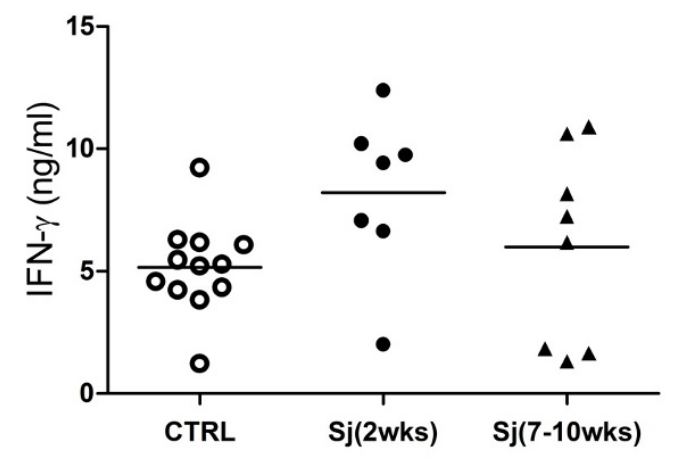

Figure 1 IL-4 production is elevated in 7 to 10 weeks Sj infected DBA/1 mice. Splenocytes from DBA/1 mice infected with Sj either for 2 weeks or 7 to 10 weeks or not infected (CTRL) were stimulated by anti-CD3 and anti-CD28 antibodies for 72 hours in vitro. The supernatants were analyzed for IL-4 (A) and IFN-g (B). Shown are the combined results from two separate experiments. Significance was tested by one-way ANOVA with Bonferroni multiple comparison test with *** and ${ }^{* *}$ as $p<0.001$ and $p<0.01$

signs of arthritis around day 35 after CII immunization (Fig 2). Injection of CII in ASCIA mice which were animals infected with the 7 weeks pre-established $S j$ infection before CIA induction, disease severity was substantially reduced as assessed by ankle swelling (Fig 2E) and cumulative clinical arthritis scores of all four limbs (Fig 2A). While control CIA animals reached a cumulative arthritis score of 9 at day 56 with incidence reaching about $85 \%$, none of the ASCIA animals developed disease (Fig 2B). In contrast, injection of CII in ESCIA mice which were animals infected with the 2 weeks pre-established $S j$ infection before CIA induction failed to provide any prophylaxis on CIA whether arthritis score (Fig 2C), arthritis incidence (Fig 2D) or clinical signs (Fig 2E) were examined. In fact, the severity of arthritis was even increased in ESCIA mice measured at day 42 (Fig 2C). As a control, non collagen-immunized mice did not develop arthritis regardless they were infected with $S j$ or not (data not shown).

\section{Anti-CII IgG and IgG2a levels were reduced in protected mice}

Anti-CII IgG has been well known for its important role in the pathogenesis of CIA and has become the marker antibody for CIA [21]. We compared the anti-CII IgG levels in protected ASCIA mice, unprotected ESCIA mice and uninfected CIA mice. As shown in Fig 3A and 3B, significantly decreased levels of anti-CII IgG were present in protected ASCIA mice as compared to uninfected CIA mice; whereas no significant difference was observed between unprotected ESCIA mice and uninfected CIA mice. This corresponded well with the prophylaxis effects observed only in ASCIA mice but not in ESCIA mice. When profiles of splenic B cells from different groups were examined, B cell percentage was found to be reduced in ASCIA mice compared to mice only challenged with CIA (36.7\% vs $47.8 \%$ ). However this reduction does not seem to explain the significantly decreased levels of anti-CII IgG found in ASCIA mice, because the total B cell number was not decreased due to the splenomegaly found in ASCIA mice. Furthermore, a more reduced percentage of $B$ cells was found in ESCIA mice compared to mice only challenged with CIA $(27.7 \%$ vs $47.8 \%$ ), though anti-CII IgG levels remained unaffected in this group.

We further compared the levels of IgG1 or IgG2a subclasses in ASCIA mice without arthritis with those from arthritic CIA or arthritic ESCIA mice. The level of IgG2a was significantly reduced in ASCIA mice comparing to CIA or ESCIA mice (Fig 3D), corresponding to lack of arthritis. In contrast, IgG1 was reduced in ESCIA mice comparing to CIA (Fig 3C), although both groups developed arthritis comparably (Fig 2). These data suggested that IgG2a was more relevant to the pathogenesis of the arthritis, and its reduction was associated with lack of arthritis as found in ASCIA mice.

\section{Protective effects in ASCIA mice were associated with enhanced production of IL-4 and IL-10 and reduced production of IFN- $\gamma$}

Many studies have indicated the importance of different Th subsets and the balance among them in the pathogenesis of arthritis in the CIA model [22]. To understand whether the alleviation of arthritis in ASCIA mice was associated with altered Th responses, splenocytes were isolated at 8 weeks after CII immunization and stimulated in vitro with anti-CD3 and anti-CD28 antibodies. The production of IFN- $\gamma$ was reduced and the production 
A
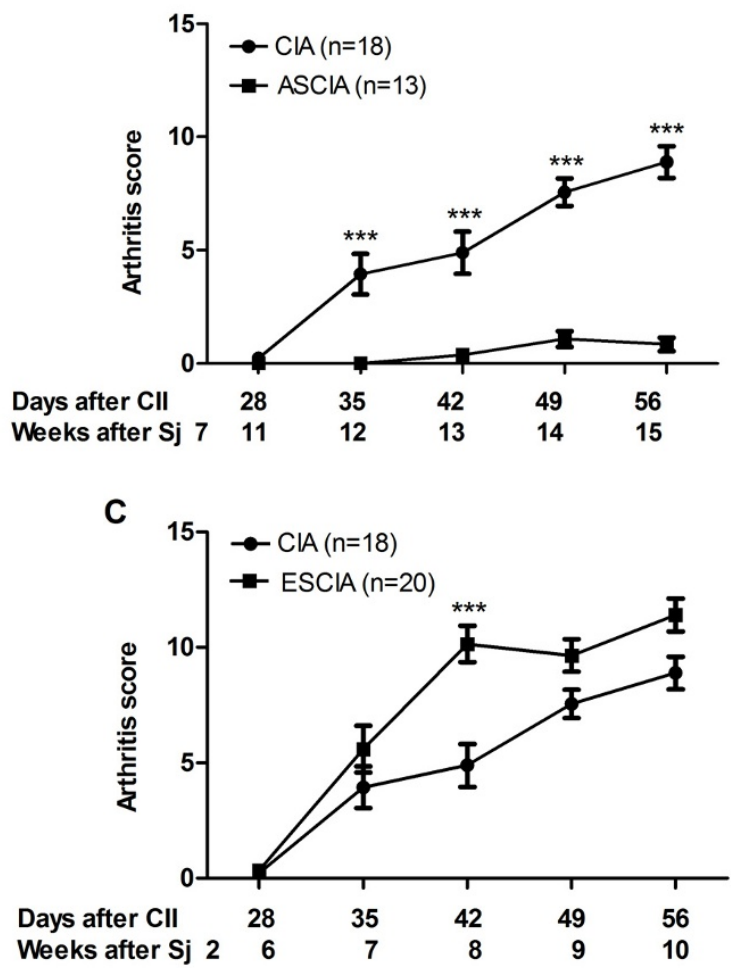

B

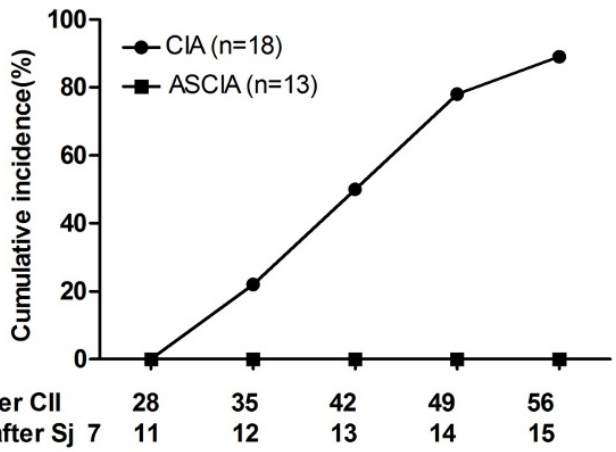

D

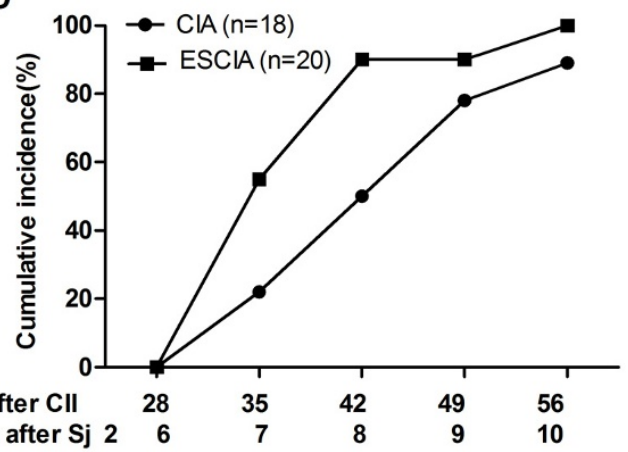

E

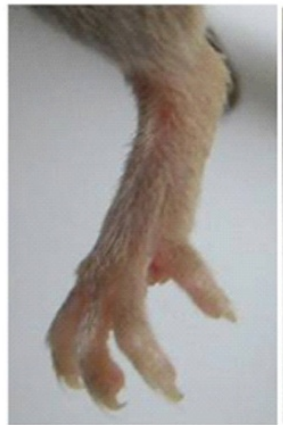

CTRL

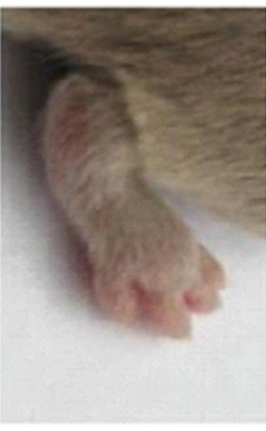

CIA

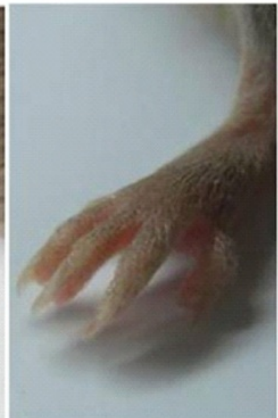

ASCIA

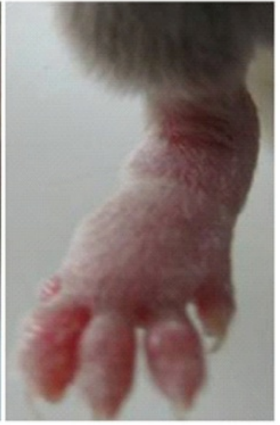

ESCIA

Figure $\mathbf{2}$ The protective effects against arthritis were only observed when a pre-established Sj infection entered acute stage. DBA/1 mice were infected with Sj for 2 weeks or 7 weeks prior to immunization with collagen II (CII) in CFA and named as ESCIA mice and ASCIA mice respectively. 7 weeks Sj infection significantly reduced the severity (A), incidence (B) and clinical signs (E) of arthritis in ASCIA mice; whereas 2 weeks infection by Sj failed to decrease the severity (C), incidence (D) and clinical signs (E) of arthritis in ESCIA mice. Arthritis was scored weekly as described in Materials and Methods. Circle and square represented uninfected CIA mice and Sj infected CIA mice respectively. CIA data represented the combined results from two different age groups from multiple experiments due to their similar severity and incidence. Data in ESCIA mice were the means and the standard errors of the mean (SEM) of clinical scores from 20 individual mice combined from two separate experiments, while data in ASCIA mice were from 13 individual mice combined from three separate experiments. Significance was tested by two way ANOVA with Bonferroni multiple comparison test with ${ }^{* *}$ as $p<0.001$. Incidence was calculated on the ratio of the number of animals with arthritis score $>=3$ on one joint over total number of mice. 
A

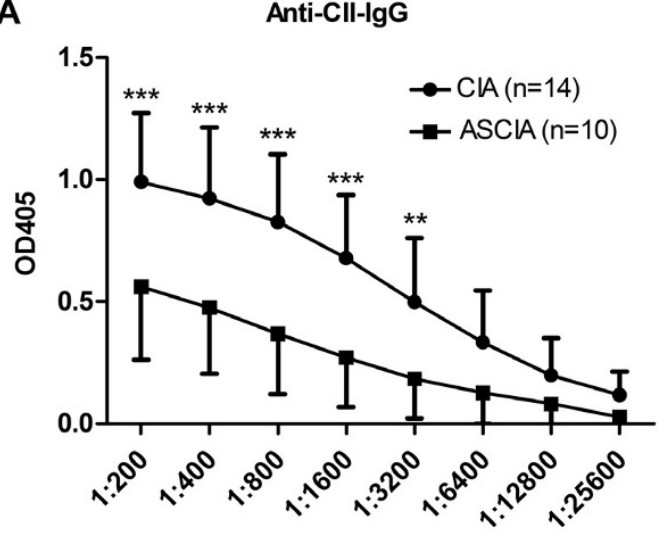

C

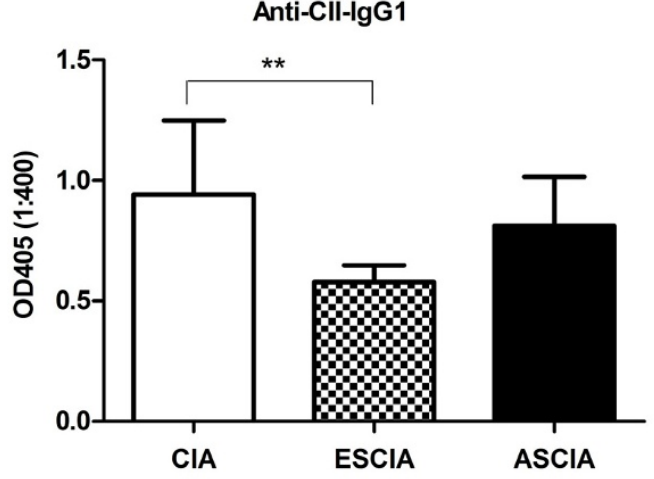

B

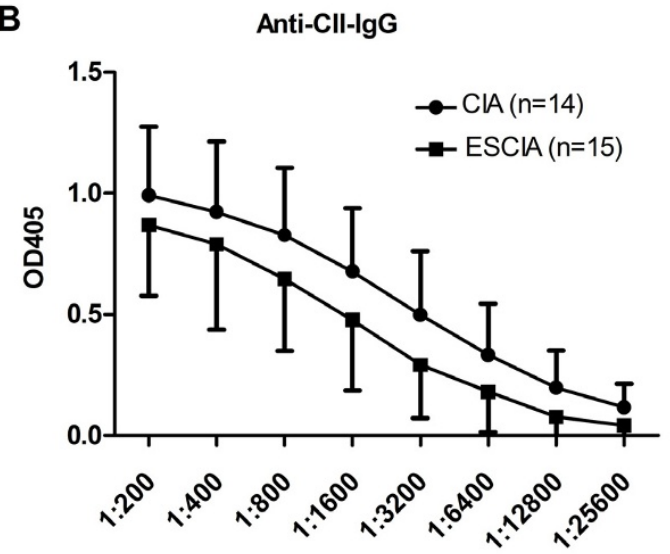

D

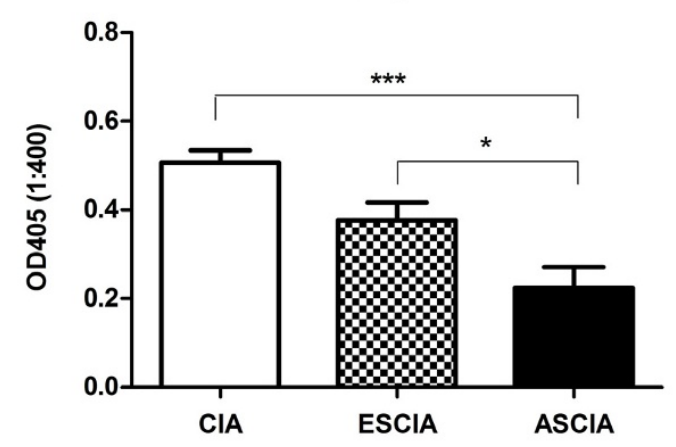

Figure 3 Anti-CII IgG and IgG2a levels were reduced in protected mice. Serums were taken from 56 days after CII challenge from ESCIA mice, ASCIA mice and CIA mice. The levels of anti-CII lgG was measured in serially diluted serum and the levels of subclasses of anti-CII lgG1 or lgG2a were measured from 1:400 diluted serum by ELISA. (A) anti-CII lgG in ASCIA vs CIA (B) anti-CII lgG in ESCIA vs CIA (C) anti-CII lgG1 (D) anti-CII lgG2a. Results were obtained from 10 to 15 serum samples collected from two or three independently performed experiments. Data presented were the mean OD \pm SEM. Asterisks*** and ${ }^{* *}$ represented $\mathrm{P}<0.001$ and $\mathrm{P}<0.01$ respectively by two way ANOVA for IgG or by one-way ANOVA for subclasses with Bonferroni multiple comparison test.

of IL-4 was enhanced in ASCIA mice compared to ESCIA and CIA mice (Fig 4A and 4B). Furthermore, moderately enhanced IL-10 levels were also found in ASCIA mice (Fig 4C). IL-17 production as induced by polyclonal stimulation of $\mathrm{T}$ cells demonstrated no significant differences between any of the groups (Fig 4D). Thus, an enhanced Th2 response and a reduced Th1 response appeared to correlate with the absence of disease.

The lack of significant IL-4 production in ESCIA mice (Fig 4A) led us to question whether the strong Th1inducing agent complete Freund's adjuvant (CFA) [23] used in CIA model would alter the induction of Th2 responses induced by schistosome infection. As shown in Fig 5, the IL-4 production was low at 2 weeks of $S j$ infection, elevated at 7 to 10 weeks of infection and then decreased after 15 weeks of infection. Thus the time course of IL-4 production by $S j$ infection in DBA/1 mice is consistent with the time course found in other strains of mice infected by $S m$ or $S j[16,18]$. However, the elevated IL-4 production occurred during 7 to 10 weeks of $S j$ infection was abrogated if CFA together with collagen was administered at 2 weeks of $S j$ infection at which IL-4 production was still low. Interestingly, CFA and collagen given at 7 weeks of infection at which IL-4 production was high prohibited the IL-4 production from being decreased when infection entered 15 week period. No differences were observed in spleen size or the appearance of the liver whether CFA was administered or not (data not shown). These results indicated that high amount of Th1-inducing agent CFA used in CIA model per se did not reverse an established Th2 response in vivo as seen in ASCIA mice which was in line with the obser- 
A

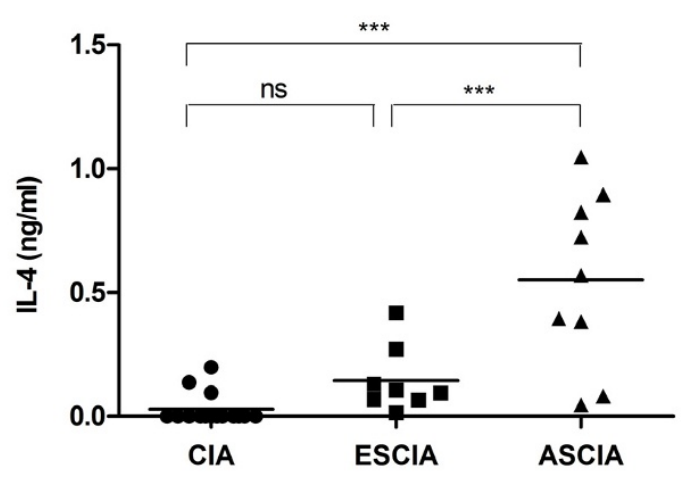

C

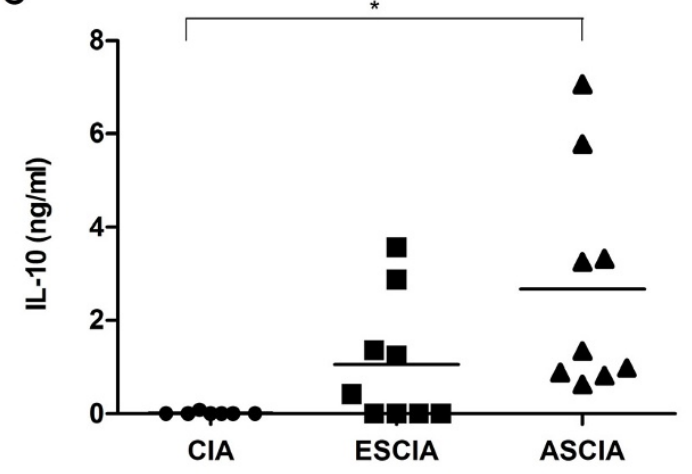

B

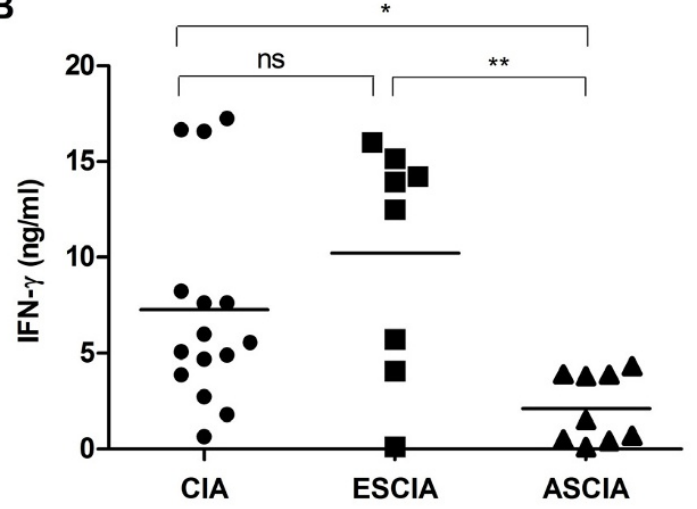

D

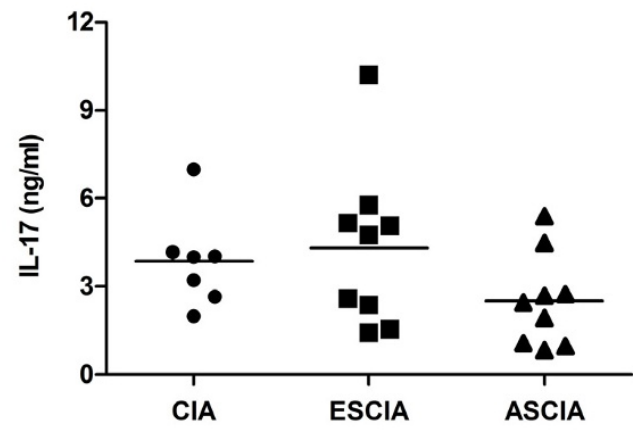

Figure 4 Protective effects in ASCIA mice were associated with enhanced production of IL-4 and IL-10 and reduced production of IFN- $\gamma$. 56 days after CII immunization in ESCIA mice, ASCIA mice and CIA mice, splenocytes were stimulated by anti-CD3 and anti-CD28 antibodies for 72 hrs. The cytokine contents for IL-4 (A), IFN-g (B), IL-10 (C) and IL-17 (D) were measured by ELISA as described in the Materials and Methods. Shown are samples from individual mouse combined from two or three separately performed experiments. Asterisks* and ${ }^{* *}$ represented $P<0.05$ and $P<0.01$ respectively by one-way ANOVA with Bonferroni multiple comparison test.

vations made in vitro that an established Th2 response was irreversible [24]. Furthermore, the ability of CFA to prevent an ongoing Th2 response from being firmly established as seen in ESCIA mice which may reflect the fact that an early phase of the Th2 response is reversible [24]. More intriguingly and significantly, presence of CFA prolonged Th2 response which otherwise would be greatly reduced seen in 15 weeks infected animals. Taken together, these results indicated that a Th2 -biased milieu present at the time when CII antigen was introduced as well as during the development of arthritis correlated with lack of arthritis in protected ASCIA animals.

\section{FoxP3 + regulatory $\mathrm{T}$ cells were not different between protected and unprotected mice}

It is widely accepted that FoxP3 ${ }^{+}$regulatory $\mathrm{T}$ cells(Treg) play critical roles in pathogenesis of various of kinds of autoimmune diseases including human RA[25,26]. Both $\mathrm{CD} 4{ }^{+} \mathrm{CD} 25^{+} \mathrm{T}$ cells and $\mathrm{CD} 4{ }^{+} \mathrm{CD} 25+\mathrm{FoxP} 3+\mathrm{T}$ cells in the spleen were measured and compared. The correspondence of these two populations of $\mathrm{T}$ cells was about 80$90 \%$ (Table 1 ). There was a slightly increased representation of FoxP3 ${ }^{+}$Treg during infection, whether it was CIA mice alone or $S j$ infection with CIA mice. However there was no remarkable increase in FoxP3 ${ }^{+}$Treg in ASCIA mice without arthritis compared to CIA or ESCIA mice with arthritis.

\section{Discussion}

Helminth parasites inhabit immune-competent hosts for long periods of time and appear to develop strategies to induce strong anti-inflammatory responses in the infected host. Loss of natural helminth exposure as a result of improved hygiene and wide-use of anti-helminth 


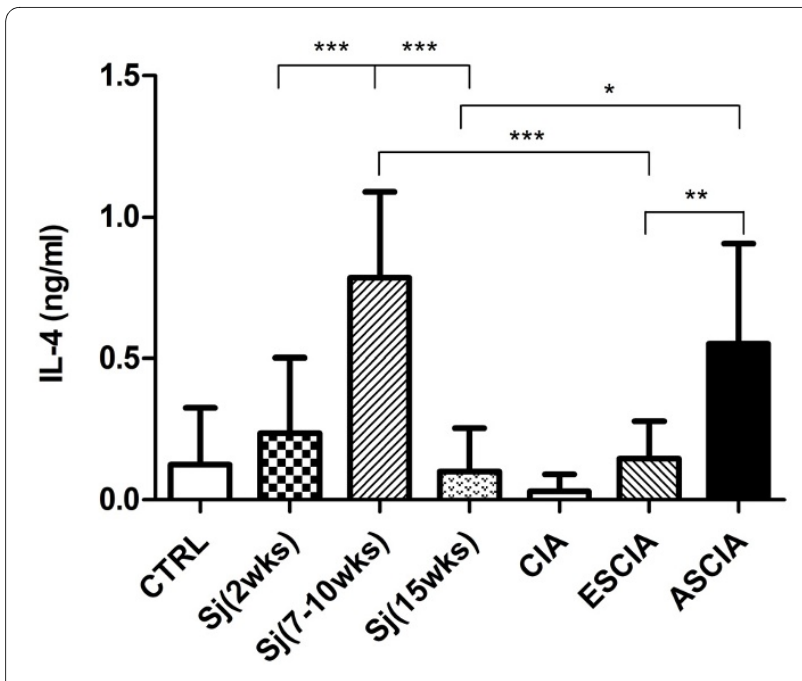

Figure 5 The effects of CFA on the IL-4 production induced by Sj infection. Splenocytes from mice of uninfected (CTRL), 2 weeks of $S j$ infection, 7 to 10 weeks of $S j$ infection, 15 weeks of $S j$ infection, CIA, ESCIA (total 10 weeks of Sjinfection), ASCIA (total 15 weeks of Sj infection) were stimulated by anti-CD3 and anti-CD28 antibodies for $72 \mathrm{hrs}$. The IL-4 contents in the supernatants were measured by ELISA. Shown are samples from individual mouse combined from two or three separately performed experiments. Asterisks* and ** represented $P<0.05$ and $\mathrm{P}<0.01$ respectively by one-way ANOVA with Bonferroni multiple comparison test.

drugs result increased incidences of immune-mediated disorders evidenced by epidemiological studies. On the other hand, the helminths provide the host with more balanced immuno-homeostasis and demonstrate beneficial effects against various kinds of autoimmune diseases in animal studies[4]. In fact several clinical trials have attempted to use helminth to treat autoimmune disease in patients and showed that exposure to helminths can reduce disease activity in patients with immune-mediated disease like ulcerative colitis[5]. In this study, we investigated the beneficial effect of schistosome infection on less-well studied collagen-induced arthritis mouse model. The beneficial effect is found to be associated with reduced anti-collagen II IgG production and with a
Th2 cytokine environment induced at egg-stage of infection.

The anti-CII antibodies have been recognized as important pathogenic factors in the initiation and development of CIA in mice. It has been reported that arthritis can be transferred passively to naive animals with CIIreactive serum [27], and by monoclonal antibody to CII [28]. In fact, a different mouse model collagen antibodyinduced arthritis (CAIA) for human RA is established by passively transferring arthritogenic antibodies against collagen. Further studies using the CAIA model found that the subclass of IgG2a appears to be more efficient in inducing RA probably due to its strong capacity to activate complement through the classical pathway [29]. The ability of inhibiting arthritis by egg-stage $S j$ infection in ASCIA mice but not by early stage infection found in our study was well correlated with reduced levels of anti-CII IgG, especially the levels of IgG2a. This alteration in class switch may reflect the significantly repressed IgG2a-promoting cytokine IFN- $\gamma$ production found in ASCIA group. On the other hand, the positive association between elevated IgG2a and the occurrence of arthritis indicates the pathogenic role played by Th1 cells and the beneficial role played by Th2 cells. Consequently, beneficial effects against CIA can only be found in the acute or egg-stage of $S j$ infection but not in early stage infection before eggs are produced in large quantity.

Schistosomiasis is a well-characterized Th2 responsedominated disease [16]. Shortly after the beginning of egg deposition, a strong egg-specific Th2 response develops characterized by high levels of IL-4, IL-5, and IL-13 which decreases when the infection enters the chronic stage $[17,30]$. Not surprisingly the negative effect of schistosome infection on the induction and development of type I diabetes in NOD model [6], multiple sclerosis in EAE model [8] and in mouse model of Graves' hyperthyroidism [10] has repeatedly been reported in mice infected by schistosme for 6-8 weeks before autoimmune diseases were induced. By comparison between the beneficial effects of $S j$ infection and the IL-4 production profiles, our study clearly demonstrated that the presence of

Table 1: CD4+CD25+FoxP3+ regulatory T Cell compartment in the spleen

\begin{tabular}{ccc}
\hline Mouse Group & CD4+CD25+ & \%CD4(SD) \\
& & CD4+CD25+FoxP3+ \\
\hline Normal & $8.9(0.3)$ & $7.4(0.9)$ \\
CIA & $10.9(2.0)$ & $9.3(3.1)$ \\
ESCIA & $10.9(1.6)$ & $7.7(0.8)$ \\
ASCIA & $12.9(2.2)$ & $10.5(1.9)$ \\
\hline
\end{tabular}

The number of mice per group is from 8 to 13 from two or three separately performed experiments. Each value was derived from the means of individual mouse in the group. 
Th2 at the beginning stage of autoimmune attack was important in conferring the protection. In addition, our study also demonstrated a positive correlation between the prolonged presence of elevated Th2 levels during the development stage of autoinflammatory response and the absence of disease.

We noticed the different result obtained by Osada et al [14] in which 2 weeks prior Schistosome infection was shown to offer protection in CIA. An obvious difference between our study and that of Osada's was that different strains of Schistosoma were used, ie, $\mathrm{Sm}$ in their study and $S j$ in ours. Since the amount of eggs along with the levels of Th2 response induced are low after 2 weeks $S m$ infection [19], Osada's study seems to suggest that the Th2 milieu present at initiation phase of autoimmune attack is not crucial to achieve the protection. In addition, elevated IL-4 production was still found to be produced by activated splenocytes from infected and CFA injected mice, suggesting that the injection of the Th1-promoting agent CFA in their study has little impact on the development of the Th2 response. It is not clear at present that if different strains of worms used can fully explain the different results obtained.

Another possible protective mechanism involved in our study was likely associated with the enhanced production of regulatory cytokine IL-10 by activated T cells found in protected animals. Th2 cells, FoxP $3{ }^{+} \mathrm{CD} 4{ }^{+}$Treg cells and FoxP3- IL-10-producing CD4+ so called Tr1 cells[31] may all contribute to this induced production of $\mathrm{T}$ cell-derived IL-10. Although no significant increase in Treg numbers was found in protected group, the possibility that the activities of Treg and Tr1 cells were enhanced can not be excluded. No significant difference on production of IL17 was found among protected mice, unprotected mice and CIA mice, even though Th17 appears to be accepted as the more critical pathogenic Th cells in the CIA model than Th1 [32].

Our study also provided an approach to explore the plasticity of Th subsets in vivo. In vitro studies have demonstrated that Th17 is an unstable subset [33], whereas Th1 and Th2 subsets, once they are well established, are not reversible [24]. With the application of Th1-promoting agent CFA at different time point, we found that CFA together with collagen is able to inhibit the Th2 response only at 2 weeks but not at 7 weeks of $S j$ infection when Th2 development is at an early stage but not well-established. Thus our results for the first time in vivo indicated that a well established Th2 response can not be reversed, whereas an early stage Th2 response can be reversed in the presence of CFA treatment. This phenomenon may serve as the basis for the beneficial effects by $S j$ infection in maintaining immune-homeostasis in infected host hence providing protection against immune-mediated diseases.

\section{Conclusion}

In summary, we demonstrated in this study that Schistosoma japonicum infection like Schistosoma masoni can inhibit the incidence and severity of autoimmune arthritis in the mouse CIA model. However this effect is absolutely infection stage-dependent, $i e$, it occurs at acute stage of infection but not at early stage of infection. Furthermore this beneficial effect is positively associated with the presence of an irreversibly established Th2 response at the initiation of autoimmune response and with its sustained high levels during the development of arthritis. Thus our study provides more insight into the complex mechanisms regarding to the "hygiene hypothesis" and the potential difficulties faced when egg or eggderived materials are used for therapeutic agents in Th1associated autoimmune diseases.

\section{Methods}

Mice

6-12 weeks old male DBA/1 mice were purchased from Songjiang Animal Facility of the Chinese Academy of Sciences of Shanghai. All mice were maintained under specific pathogen-free conditions and fed with standard laboratory food and water. All procedures performed on animals within this study were conducted in accordance with and by approval of the Internal Review Board of Tongji University School of Medicine.

\section{Schistosoma japonicum Infection in mice}

DBA/1 mice were infected by percutaneous exposure of the abdomen with 20 cercariae per mouse. Cercariae of $S j$ used for all experiments were obtained from Oncomelania hupensis snails collected from Guichi County, Anhui Province, and maintained at the National Institute of Parasitic Diseases, Shanghai. Uninfected animals of the same sex and age were maintained as controls. At the end of each experiment, the livers and spleens from infected mice were routinely examined to ensure that schistosomiasis was established.

\section{Induction and assessment of $\mathrm{CIA}$}

CIA was induced according to the methods as described by Current Protocol in Immunology [34]. Chicken type II collagen(CII) (Sigma) was dissolved in $0.05 \mathrm{M}$ acetic acid at concentration of $2 \mathrm{mg} / \mathrm{ml}$ by stirring overnight at $4^{\circ} \mathrm{C}$ and was then emulsified in an equal volume of complete Freund's adjuvant (CFA) containing $4 \mathrm{mg} / \mathrm{ml}$ Mycobacterium tuberculosis (Chondrex, Redmond, WA). Male DBA/1 mice were injected intradermally at the base of the tail with $0.1 \mathrm{ml}$ of emulsion containing $100 \mu \mathrm{g}$ of collagen II. 21 days after primary immunization, mice were boosted with $0.1 \mathrm{ml}$ of the mixture of $1 \mathrm{mg} / \mathrm{ml} \mathrm{CII} \mathrm{emul-}$ sified in incomplete Freund's adjuvant (Sigma) via the same route. In order to keep the age of animals compara- 
ble for arthritis profiles, different ages of mice were selected for experiments for either early or acute infection. For experiments of early stage of infection, mice of 10-12 weeks old were used with first injection of CII at 2 weeks after $S j$ infection, named ESCIA mice. For experiment of acute stage of infection, mice of 6-8 weeks old were used with first injection of CII at 7 weeks after $S j$ infection, named ASCIA mice.

Arthritis was scored as described in Current Protocol in Immunology [34] from day 21 using a scale of 0-4 per limb in which $0=$ No evidence of redness and swelling; 1 $=$ Redness and mild swelling confined to the mid-foot or ankle joint; 2 = Redness and mild swelling extending from the ankle to the mid-foot; 3 = Redness and moderate swelling extending from the ankle to the metatarsal joints; $4=$ Redness and severe swelling encompass the ankle, foot and digits. The total arthritis score in one mouse is the sum of scores of all four feet with maximum score of 16. The incidence of CIA was calculated based on the number of mice with at least one foot scored higher than 3.

\section{Measurement of antibodies against CII}

The levels of anti-CII IgG or its subclasses in serum were measured by ELISA. Serum was collected at day 56 after first CII immunization. In brief, 96-well ELISA microplates (Greiner) were coated with CII at $5 \mu \mathrm{g} / \mathrm{ml}$ dissolved in $0.1 \mathrm{~N} \mathrm{NaHCO3,} \mathrm{pH9.6} \mathrm{buffer} \mathrm{at} 100 \mu \mathrm{l} /$ well at $4^{\circ} \mathrm{C}$ overnight. $100 \mu \mathrm{l}$ of diluted serum sample was incubated at $4^{\circ} \mathrm{C}$ overnight. The plates were washed with PBST (0.5\% Tween-20 in PBS) three times, followed by adding peroxidase conjugated goat anti-mouse IgG at 1:2000 (CapitalBo Corp., Beijing) or peroxidase-conjugated anti-mouse IgG1 or anti-mouse IgG2a at 1:10000 (Santa Cruz) at $100 \mu \mathrm{l} /$ well. After $2 \mathrm{hr}$ incubation at room temperature and wash, the final color development was achieved by adding peroxidase substrate ABTS (2,2'Azino-bis(3-Ethylbenzthiazoline-6-Sulfonic Acid) Sigma) to each well at $100 \mu \mathrm{l} /$ well and the absorbance was measured at $405 \mathrm{~nm}$ at appropriate time.

\section{Measurement of cytokine production by splenocytes}

To study cytokine expression by $\mathrm{T}$ lymphocytes, isolated spleen cells $\left(5 \times 10^{6}\right.$ cells/well $)$ from mice that were immunized by CII for 8 weeks or from control mice were stimulated with $1 \mu \mathrm{g} / \mathrm{ml}$ anti-CD3e (eBioscience) and 1 $\mu \mathrm{g} / \mathrm{ml}$ anti-CD28 (eBioscience) at $37^{\circ} \mathrm{C}, 5 \% \mathrm{CO} 2$ in $10 \%$ FCS/RPMI(GIBCO) culture medium for $72 \mathrm{hrs}$. The cytokine contents in the supernatants were analyzed by ELISA analysis as described by the e-Bioscience protocol http://www.ebioscience.com. All coating and detection antibodies were purchased from e-Bioscience (Dakewe, Shanghai), and recombinant mouse cytokines were obtained from Peprotech (Dakewe, Shanghai) for standard curves in ELISA assay.

\section{Flow cytometric analysis of regulatory $\mathrm{T}$ cell, $\mathrm{T}$ cell and B \\ cell}

The levels of regulatory $\mathrm{T}$ cell (Treg) in splenocytes were measured by flowcytometry analysis as instructed by the Mouse Regulatory T cell Staining Kit (eBioscience). T cell and $\mathrm{B}$ cell were detected by FACS analysis staining with either FITC-anti-CD3e (eBioscience) or PE-anti-B220 (eBioscience). All data were collected on a FACSCalibur (Becton Dickinson) and analyzed with FlowJo software (Tree Star, Ashland, OR).

\section{Statistical analyses}

Differences in arthritis score and differences in antibody levels of anti-CII IgG were analyzed with two-way ANOVA with Bonferroni multiple comparison tests. Differences in levels of cytokine production and subclasses of IgG were analyzed by one-way ANOVA with Bonferroni multiple comparison test. Calculations for statistical analysis were performed with Prism software. $\mathrm{P}<0.05\left(^{*}\right)$, $\mathrm{P}<0.01(* *)$ and $\mathrm{P}<0.001\left({ }^{* * * *}\right)$ were considered as statistically significant.

\section{Abbreviations}

Sj: Schistosoma japonicum; Sm: Schistosoma masoni; Cll: type II collagen; CIA collagen-induced arthritis; CFA: complete Freund's adjuvant; ELISA: enzyme linked immunosorbant assay; IFN: interferon; IL: interleukin.

\section{Authors' contributions}

$Y K H, J L, Y L$ and XPC participated in the design of the study. $J L$ did the initial work and YKH performed majority of the experiments. CXC, FLC and YSB recorded the arthritis. JL and WJZ did the infection. YKH and XPC participated in the statistical analysis. XPC wrote the manuscript. All authors read and approved the final manuscript.

\section{Acknowledgements}

This work was supported by a grant from the National Basic Research Program (973 program) (no.2007CB513100) and a grant from Shanghai Science and Technology Key Program (064119619) awarded to XPC. We thank Dr. Nina Lear Markham for reviewing and editing the manuscript.

\section{Author Details}

1Department of Immunology, Tongji University School of Medicine, 1239 Siping Road, Shanghai 200092, China and 2Department of Pathogen Biology, Tongji University School of Medicine, 1239 Siping Road, Shanghai 200092, China

Received: 28 January 2010 Accepted: 10 June 2010

Published: 10 June 2010

\section{References}

1. EAS Group: Variation and trends in incidence of childhood diabetes in Europe. Lancet 2000, 355:873-6.

2. Farrokhyar F, Swarbrick ET, Irvine EJ: A critical review of epidemiological studies in inflammatory bowel disease. Scand J Gastroenterol 2001, 36:2-15.

3. Strachan DP: Hay fever, hygiene, and household size. Bmj 1989, 299:1259-60

4. Dunne DW, Cooke A: A worm's eye view of the immune system: consequences for evolution of human autoimmune disease. Nat Rev Immunol 2005, 5:420-6. 
5. Elliott DE, Summers RW, Weinstock JV: Helminths as governors of immune-mediated inflammation. Int J Parasitol 2007, 37:457-64.

6. Cooke A, Tonks P, Jones FM, O'Shea H, Hutchings P, Fulford AJ, Dunne DW: Infection with Schistosoma mansoni prevents insulin dependent diabetes mellitus in non-obese diabetic mice. Parasite Immunol 1999, 21:169-76.

7. Zaccone P, Burton O, Miller N, Jones FM, Dunne DW, Cooke A: Schistosoma mansoni egg antigens induce Treg that participate in diabetes prevention in NOD mice. Eur J Immuno/ 2009, 39:1098-107.

8. La Flamme AC, Ruddenklau K, Backstrom BT: Schistosomiasis decreases central nervous system inflammation and alters the progression of experimental autoimmune encephalomyelitis. Infect Immun 2003, 71:4996-5004

9. Zheng X, Hu X, Zhou G, Lu Z, Qiu W, Bao J, Dai Y: Soluble egg antigen from Schistosoma japonicum modulates the progression of chronic progressive experimental autoimmune encephalomyelitis via Th2-shift response. J Neuroimmunol 2008, 194:107-14

10. Nagayama Y, Watanabe K, Niwa M, McLachlan SM, Rapoport B: Schistosoma mansoni and alpha-galactosylceramide: prophylactic effect of Th1 Immune suppression in a mouse model of Graves' hyperthyroidism. J Immunol 2004, 173:2167-73.

11. HM Mo, WQ Liu, JH Lei, Cheng YL, Wang CZ, YL Li: Schistosoma japonicum eggs modulate the activity of CD4+CD25+ Tregs and prevent development of colitis in mice. Exp Parasitol 2007, 116:385-9.

12. Mangan NE, van Rooijen N, McKenzie AN, Fallon PG: Helminth-modified pulmonary immune response protects mice from allergen-induced airway hyperresponsiveness. J Immunol 2006, 176:138-47.

13. Mattsson L, Larsson P, Erlandsson-Harris $H$, Klareskog L, Harris RA: Parasite-mediated down-regulation of collagen-induced arthritis (CIA) in DA rats. Clin Exp Immunol 2000, 122:477-83.

14. Osada Y, Shimizu S, Kumagai T, Yamada S, Kanazawa T: Schistosoma mansoni infection reduces severity of collagen-induced arthritis via down-regulation of pro-inflammatory mediators. Int J Parasitol 2009, 39:457-64.

15. Pearce EJ, MacDonald AS: The immunobiology of schistosomiasis. Nat Rev Immunol 2002, 2:499-511.

16. Grzych JM, Pearce E, Cheever A, Caulada ZA, Caspar P, Heiny S, Lewis F, Sher A: Egg deposition is the major stimulus for the production of Th2 cytokines in murine schistosomiasis mansoni. J Immunol 1991, 146:1322-7.

17. MJ Ji, Su C, Wang Y, Wu HW, Cai XP, Li GF, Zhu X, Wang XJ, Zhang ZS, Wu $\mathrm{GL}$ : Characterization of $\mathrm{CD} 4+\mathrm{T}$ cell responses in mice infected with Schistosoma japonicum. Acta Biochim Biophys Sin (Shanghai) 2006, 38:327-34.

18. YH Xu, Macedonia J, Sher A, Pearce E, Cheever AW: Dynamic analysis of splenic Th1 and Th2 lymphocyte functions in mice infected with Schistosoma japonicum. Infect Immun 1991, 59:2934-40.

19. Pearce EJ, Caspar P, Grzych JM, Lewis FA, Sher A: Downregulation of Th1 cytokine production accompanies induction of Th2 responses by a parasitic helminth, Schistosoma mansoni. J Exp Med 1991, 173:159-66.

20. Takagi N, Mihara M, Moriya Y, Nishimoto N, Yoshizaki K, Kishimoto T, Takeda Y, Ohsugi Y: Blockage of interleukin- 6 receptor ameliorates joint disease in murine collagen-induced arthritis. Arthritis Rheum 1998 , 41:2117-21.

21. Rowley MJ, Nandakumar KS, Holmdahl R: The role of collagen antibodies in mediating arthritis. Mod Rheumatol 2008, 18:429-41.

22. Sarkar S, Cooney LA, White P, Dunlop DB, Endres J, Jorns JM, Wasco MJ, Fox DA: Regulation of pathogenic IL-17 responses in collagen-induced arthritis: roles of endogenous interferon-gamma and IL-4. Arthritis Res Ther 2009, 11:R158.

23. Billiau A, Matthys P: Modes of action of Freund's adjuvants in experimental models of autoimmune diseases. J Leukoc Biol 2001, 70:849-60.

24. Grogan JL, Mohrs M, Harmon B, Lacy DA, Sedat JW, Locksley RM: Early transcription and silencing of cytokine genes underlie polarization of $T$ helper cell subsets. Immunity 2001, 14:205-15.

25. Curotto de Lafaille MA, Lafaille JJ: Natural and adaptive foxp3+ regulatory T cells: more of the same or a division of labor? Immunity 2009, 30:626-35.

26. Esensten JH, Wofsy D, Bluestone JA: Regulatory T cells as therapeutic targets in rheumatoid arthritis. Nat Rev Rheumatol 2009, 5:560-5.
27. Stuart JM, Dixon FJ: Serum transfer of collagen-induced arthritis in mice. J Exp Med 1983, 158:378-92.

28. Holmdahl R, Rubin K, Klareskog L, Larsson E, Wigzell H: Characterization of the antibody response in mice with type II collagen-induced arthritis, using monoclonal anti-type II collagen antibodies. Arthritis Rheum 1986, 29:400-10.

29. Nandakumar KS, Johansson BP, Bjorck L, Holmdahl R: Blocking of experimental arthritis by cleavage of IgG antibodies in vivo. Arthritis Rheum 2007, 56:3253-60.

30. Chiaramonte MG, Schopf LR, Neben TY, Cheever AW, Donaldson DD, Wynn TA: IL-13 is a key regulatory cytokine for Th2 cell-mediated pulmonary granuloma formation and IgE responses induced by Schistosoma mansoni eggs. J Immunol 1999, 162:920-30.

31. Barnes MJ, Powrie F: Regulatory T cells reinforce intestinal homeostasis. Immunity 2009, 31:401-11

32. Nakae S, Nambu A, Sudo K, Iwakura Y: Suppression of immune induction of collagen-induced arthritis in IL-17-deficient mice. J Immuno/ 2003, 171:6173-7.

33. Lee YK, Turner H, Maynard CL, Oliver JR, Chen D, Elson CO, Weaver CT: Late developmental plasticity in the Thelper 17 lineage. Immunity 2009, 30:92-107.

34. Rosloniec E, Cremer M, Kang A, Myers LK: Collagen-Induced Arthritis. In current protocols in immunology John Wiley \& Sons, Inc; 1996:15.5.1-15.5.24

doi: 10.1186/1471-2172-11-28

Cite this article as: He et al., The inhibitory effect against collagen-induced arthritis by Schistosoma japonicum infection is infection stage-dependent BMC Immunology 2010, 11:28

\section{Submit your next manuscript to BioMed Central and take full advantage of:}

- Convenient online submission

- Thorough peer review

- No space constraints or color figure charges

- Immediate publication on acceptance

- Inclusion in PubMed, CAS, Scopus and Google Scholar

- Research which is freely available for redistribution
C) BioMed Central 